\title{
Cigarette Smoke Induce Alteration of Structure and Function in Alveolar Macrophages
}

\author{
Yuriko Hirono, Ayaka Kawazoe, Masahiko Nose, Masaaki Sakura, and Minoru Takeuchi
}

\begin{abstract}
Cigarette smoke (CS) is released into the atmosphere, and impact lung health in non-smoker but not smoker. CS is inhaled into the lung by respiration and affects alveolar macrophages (AM). AM play an important role of immune system in the lung. In this study, we investigated the effect of CS on DNA damage and immune function in AM. The number of AM was significantly increased in CS exposed mice compared with non CS-exposed mice. Expressions of CD11b, TLR-2 and CD14 on AM were significantly inhibited in CS exposed mice but not CD16. Phagocytic activity of AM was significantly inhibited in CS exposed mice. Both of tail moment and tail length of AM as indicator of DNA damage were significantly increased in CS exposed mice. CS was a risk factor for DNA damage of $A M$ and induced inhibition of immunological functions in AM mediated with DNA damage. These results suggest that changes of intracellular structure, inhibition of phagocytosis and TLR expression and induced-DNA damage of AM by CS may result in easily infection of bacteria or virus and carcinogenesis.
\end{abstract}

Index Terms-Cigarette smoke, alveolar macrophages, DNA damage, phagocytic activity

\section{INTRODUCTION}

Cigarette smoke (CS) is an important factor in environment because CS is released to environment in out-door and in-door. It is generally accepted that prolonged exposure to cigarette smoke is associated with an increased prevalence of a variety of respiratory disease [1], the mechanism of which have not been clearly defined. CS is released into the atmosphere, and impact lung health in non-smoker but not smoker. Immune system has long been known to play an essential role in defense mechanisms against infectious disease. Previous studies have shown cigarette tobacco smoke can impair pulmonary immune function and hence alter resistance to the development of lung disease [2]. Alveolar macrophages (AM) play a major role in lung immune system. Functions of AM are largely classified into phagocytosis, secretion (cytokines, enzymes and reactive oxygen intermediates) and antigen presentation [3]. CS is inhaled into the lung by respiration and affects AM. In previous studies, we have demonstrated that cigarette tobacco smoking induces functional changes in AM [4]. However, the influence of cigarette smoke on the AM functions is not yet fully understood. Therefore, we investigated the effect of cigarette smoking on the phagocytosis, surface antigens associated with immune

Manuscript received November 15, 2012; revised January 10, 2013.

Yuriko Hirono and Minoru Takeuchi are with the Faculty of Life Sciences, Kyoto Sangyo University, Kyoto, Japan (e-mail: s4373@cc.kyoto-su.ac.jp, mtakex@cc.kyoto-su.ac.jp). functions and DNA damage in AM.

\section{MATERIALS AND METHODS}

\section{A. Animals}

We purchased 8-week-old C57BL/6 female mice from Japan SLC, Inc. (Shizuoka, Japan) and quarantined them for at least for 3 days prior to their exposure to cigarette smoke (CS). Mice were housed in transparent plastic cages with stainless wire lids in the animal facility of Kyoto Sangyo University (Kyoto, Japan). Mice were maintained under standard conditions, with a dark period from $8 \mathrm{pm}$ to $8 \mathrm{am}$, and water and food were provided ad libitum before, during and after exposure. Mice were used between 8 to 10 weeks of age at the time of exposure. This study was approved by the Kyoto Sangyo University committee for animal care and welfare.

\section{B. Cigarette Smoke (CS) Exposure}

Mice in the CS-exposed group $(S, n=10)$ were exposed to mainstream smoke of 20 filter-tipped cigarettes (Reference Cigarette CORESTA APPROVED MONITOR No.6) per day during 10 days using a Hamburg II smoking machine (Leybold-Heraeus, Hamburg, Germany). CS was diluted with air at a ratio of $7: 3$, and the puff volume was 35 $\mathrm{ml} / 2 \mathrm{sec} / 1$ puff. Mice in control group (non-smoked group: NS, $n=10$ ) were exposed to air instead of CS under identical conditions as the CS group.

\section{Blonchoalveolar Lavage (BAL)}

Mice were sacrificed under anesthetic the day after the last CS exposure. BAL was performed by injection of phosphate-buffered saline (PBS) through intra-trachea, and then BAL fluids were collected. Recovered cells in BAL fluids were separated by centrifugation $(220 \times \mathrm{g}, 10$ minutes, $4^{\circ} \mathrm{C}$ ), and resuspended in culture medium (Roswell Park Memorial Institute medium [RPMI1640, Nacalai tesque, Kyoto, Japan] supplemented with $1 \%$ fetal calf serum [FCS, Hyclone laboratories, UT, USA], $50 \mathrm{mM}$ L-glutamine [Nacalai tesque], $100 \mu \mathrm{g} / \mathrm{ml}$ streptomycin [Meiji Seika, Tokyo, Japan], and $100 \mathrm{U} / \mathrm{ml}$ penicillin [Meiji Seika]). Number and viability of recovered cells were determined by $0.2 \%$ trypan blue exclusion test, and viability was found to be more than $98 \%$. AM collected from the BAL were more than $95 \%$ pure, as determined by morphology and nonspecific esterase staining. Intracellular structure of AM was analyzed by Dot plot analysis using Fluorescence Activated Cell Sorter (FACS). Morphology of AM was observed by Giemsa staining under light microscope. 


\section{Surface Antigens Expression Associated with Phagocytosis}

AM $\left(5 \times 10^{4}\right.$ cells $\left./ 100 \mu \mathrm{l}\right)$ were resuspended in FACS buffer (PBS containing $100 \mu \mathrm{g} / \mathrm{ml} \mathrm{CaCl}_{2} / \mathrm{MgCl}_{2}, 0.01 \%$ sodium azide and $1 \% \mathrm{FCS}$ ), and stained with $0.5 \mu \mathrm{g}$ of fluorescein isothiocyanate (FITC) or phycoerythrin (PE)-conjugated monoclonal antibodies $(\mathrm{mAb})$ at $4{ }^{\circ} \mathrm{C}$ for 45 minutes. FITC-conjugated anti-CD11b, anti-Toll like receptor (TLR)-2, anti-CD16 and PE-conjugated anti-CD14 $\mathrm{mAb}$ were obtained from BD Bioscience. After incubation, AM were washed twice with FACS buffer and then resuspended in $300 \mu \mathrm{l}$ of FACS buffer. Ten thousand cells were acquired and gated appropriately by Dot plot using forward and side scatter. Surface antigens-positive cells were analyzed using FACS Calibur (Becton Dickinson, CA, USA).

\section{E. Phagocytic Activity}

AM $\left(5 \times 10^{4}\right.$ cells $\left./ 100 \mu \mathrm{l}\right)$ were mixed with FITC-labeled latex beads and cultured at $37^{\circ} \mathrm{C}$ under the presence of $5 \%$ $\mathrm{CO}_{2}$ for 2 hours. After 2 hours, AM were centrifuged at $185 \times \mathrm{g}$ for 10 minutes, and were resuspended in $300 \mu \mathrm{l}$ of FACS buffer. Five thousand cells were acquired and gated appropriately by Dot plot using forward and side scatter. Phagocytic activity of analyzed using FACS Calibur.

\section{F. DNA Damage}

Analysis of DNA damage was performed using CometAssay kit (Trevigen, MD, USA) according to the manufacture's protocol. Briefly, AM were combined with molten LMAgarose solution including 1\% low melting agarose in PBS at $42{ }^{\circ} \mathrm{C}$, and immediately pipette onto CometSlide and left at $4{ }^{\circ} \mathrm{C}$ for 10 minutes. The slides were immersed in pre-chilled Lysis Solution $(2.5 \mathrm{M} \mathrm{NaCl}, 100$ $\mathrm{mM}$ EDTA, $10 \mathrm{mM}$ Tris- $\mathrm{HCl}, \mathrm{pH} 10,1 \%$ sodium lauryl sarcosinate, and $1 \%$ Triton $\mathrm{X}-100$ ) for 60 minutes, and then left in Alkali Solution which contains $1.2 \% \mathrm{NaOH}$ in $1 \mathrm{mM}$ EDTA for 40 minutes. Slides were washed with $1 \times \mathrm{TBE}$ electrophoresis buffer, and electrophoresis was conducted for 10 minutes at 1 volt per $\mathrm{cm}$ (measured electrode to electrode). Slides were then air dry and fixed with ethanol. For analysis, Slides were stained with SYBR green and observed under fluorescence microscopy (Olympus Co, Tokyo, Japan) with excitation at $494 \mathrm{~nm}$ and emission at $521 \mathrm{~nm}$. Image analysis was performed using Comet Analyzer Software (Youworks Co., Tokyo, Japan). DNA damages were quantified by measuring for increase in tail moment and tail length as indicator of DNA damage.

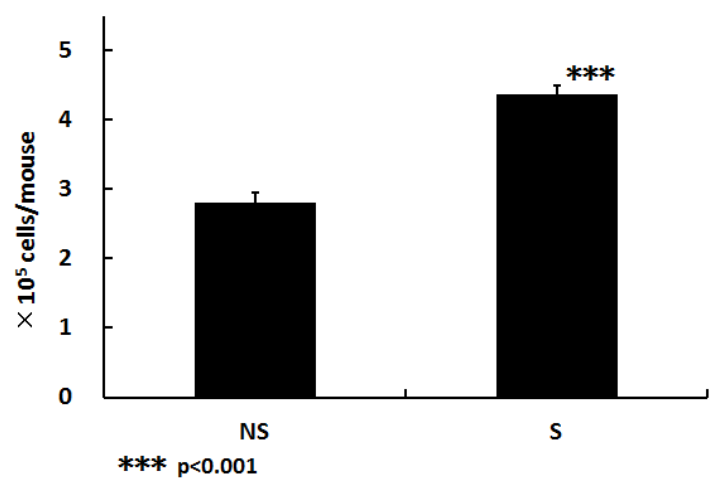

Fig. 1.The effect of CS on the number of AM.

\section{G. Statistics}

Data are presented as means \pm standard error (SE). Comparisons among treatment groups were made with the Student's t test following two-way analysis. Differences at $p<0.05$ were considered significant.

\section{RESULTS AND DISCUSSIONS}

\section{A. Effect of CS on the Number and Dot Plot of AM}

Cigarette smoking exerts a great influence on the immune system. For example cigarette smokers have increased total white blood cell count, decreased CD4/CD8 T cell ratios, decreased serum immunoglobulin levels and suppressed natural killer activity [5], [6]. Alveolar macrophages (AM) play a major role in lung defense mechanisms. Cigarette smoking induces various changes in functions of AM [7]. The number of AM was significantly increased in $\mathrm{S}\left(4.4 \times 10^{5}\right.$ cells/mouse) compared with NS $\left(2.8 \times 10^{5}\right.$ cells/mouse) (Fig. 1 ). Both of forward scatter and side scatter values by Dot plot analysis were increased in S compared with NS (Fig. 2), which indicates that CS caused AM to increase in size with corresponding enlargement of internal cell structures. AM were enlarged and vacuoles were appeared in cytoplasm in S (Fig. 3). These alterations may be due to intake of CS particles by AM.

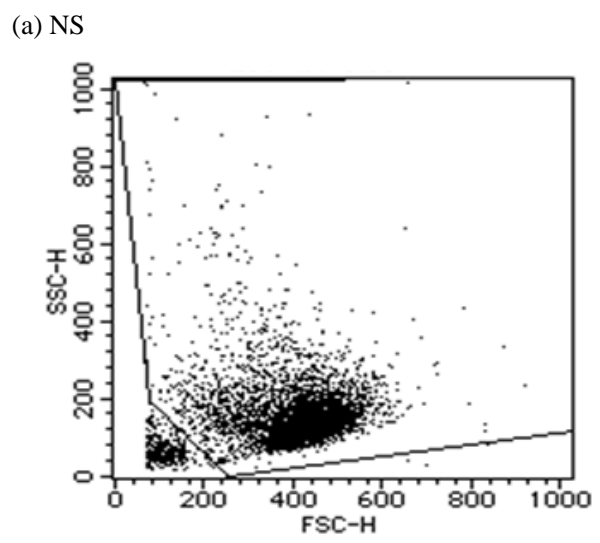

(b) $\mathrm{S}$

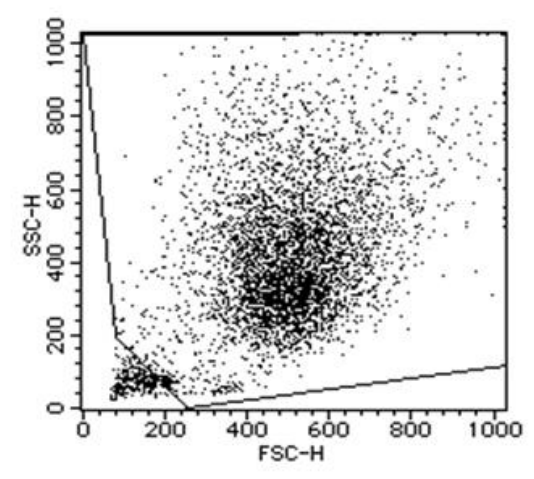

Fig. 2. The effect of CS on Dot Plot of AM.

\section{B. Effect of CS on Surface Antigens Associated with Phagocytic Activity and Recognition of Microorganism in AM}

Surface antigens associated with phagocytosis on AM were also studied. CD11b is a receptor for C3b complement, and CD16 is a receptor for Fc of $\mathrm{IgG}$. These surface receptors 
assist phagocytosis of AM by acting as an opsonin. The percentage of CD11b, CD14 and TLR-2 antigen-positive cells was significantly $(p<0.01)$ decreased in $\mathrm{S}$ compared with NS. However, the percentage of CD16 antigen-positive cells was not different between S and NS (Fig. 4). These results suggest that the inhibition of phagocytic activity by CS was caused by the inhibition of CD11b surface antigen related with $\mathrm{C} 3 \mathrm{~b}$ complement receptor of opsonin. Decreased CD11b antigen-positive cells from CS exposed mice have previously been reported [8].

(a) NS

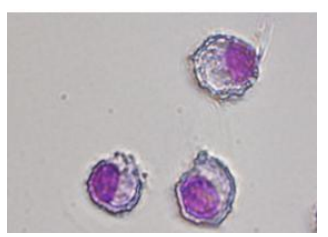

(b) $\mathrm{S}$

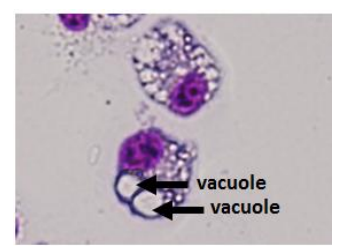

Fig. 3. The effect of CS on morphology AM.

\section{Effect of CS on Phagocytic Activity of AM}

The phagocytic activity of AM was significantly $(p<0.001)$ decreased in S compared with NS (Fig. 5). The inhibition of phagocytosis in AM may be due to CS particles saturating the normal phagocytic ability of AM. There are reports that AM from CS exposed mice had inhibited phagocytosis to C.albicans and latex beads [9], [10]. These results agree with the phagocytic activity seen in our present report.

\section{Effect of CS on DNA Damage in AM}

Both of tail moment and tail length of AM as indicator of DNA damage were significantly $(p<0.001)$ increased in S. (Fig. 6). It has previously been reported that CS increased reactive oxygen species (ROS) generation in AM [11], [12]. CS-induced DNA damage may cause by excess of ROS production. Takeuchi et al. have suggested the relationship between intracellular ROS and induced oxidative DNA damage in human neutrophil-like cells [13]. Our data is similar to a prior report that CS or CS extract induces DNA damage in human lung tissue and B-lymphoblastoid cell line MCL-5[14], [15], human bronchial epithelial cells and lung fibroblasts without leading to apoptosis or necrosis [16], [17]. Accordingly, it is considered that CS-induced DNA damage of AM may lead to altered cellular functions and affect the pulmonary immune system.

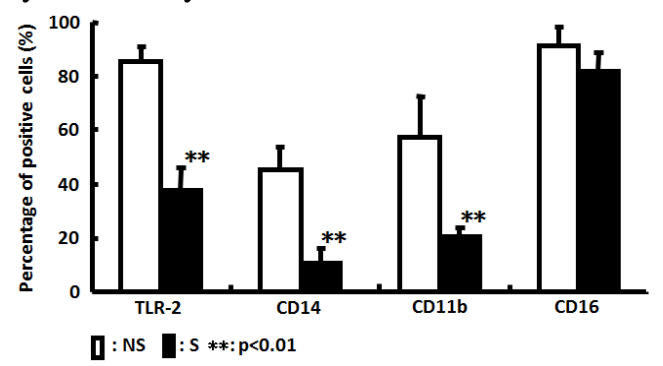

Fig. 4. The effect of CS on surface antigen of A

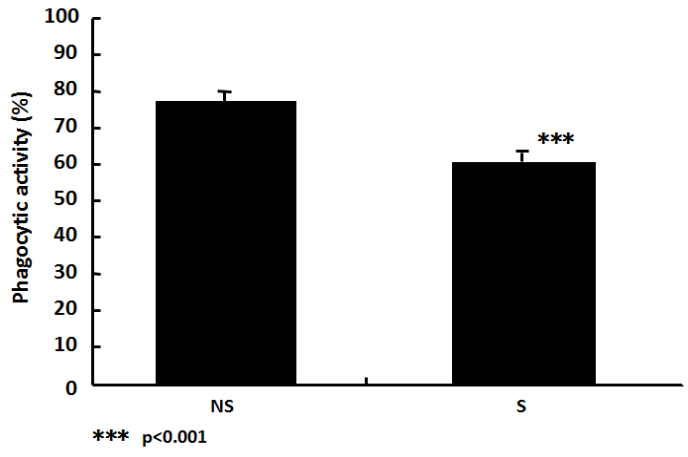

Fig. 5. The effect of CS on phagocytic activity of AM.

(a) Tail moment

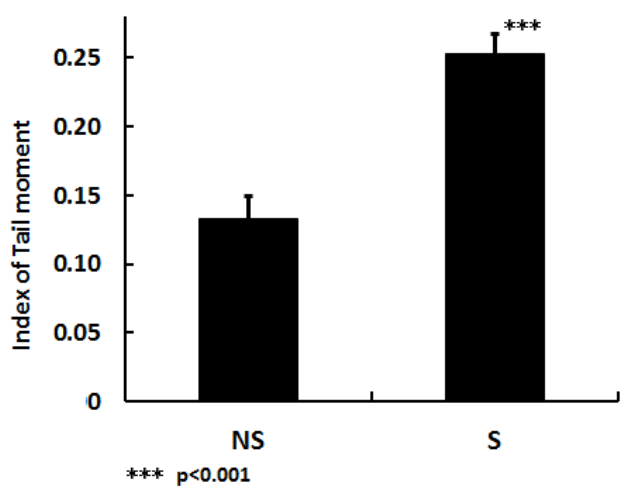

(b) Tail length

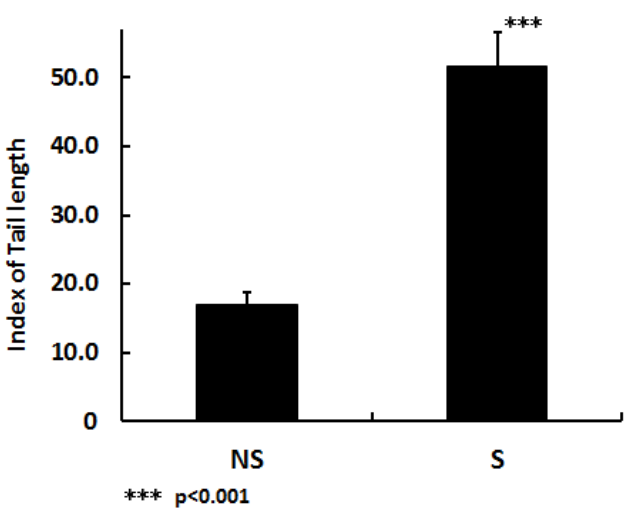

Fig. 6. The effect of CS on DNA damage of AM.

\section{CONCLUSION}

We investigated the effect of CS on AM. CS exposure caused alteration of cell size and intracellular structure in AM. CS induced significantly increase of DNA damage in AM. Phagocytic activity of AM was significantly decreased by CS. Expressions of CD11b, TLR-2 and CD14 on AM were decreased by CS, but not CD16. CS was a risk factor for DNA damage of AM and inhibited immunological functions of AM mediated with DNA damage. These results suggest that alteration of intracellular structure, inhibited phagocytic activity and expressions of surface antigens associated with phagocytosis and induced-DNA damage of AM by CS may result in easily infection of bacteria or virus and carcinogenesis.

\section{ACKNOWLEDGMENT}

The authors would to express their gratitude to Japan 
Society for the Promotion of Science for the financial support under the Grants-in-Aid for Scientific Research(C) 23500826.

\section{REFERENCES}

[1] G. S. Maritz and M. Mutemwa, "Tobacco smoking: patterns, health consequences for adults, and the long-term health of the offspring," Glob. J. Health Sci., vol. 4, no. 4, pp. 62-5, May 2012.

[2] M. Sopori, "Effects of cigarette smoke on the immune system," Nat. Rev. Immunol., vol. 2, no. 5, pp. 372-377, May 2002.

[3] O. Fels and Z. A. Cohn, "The alveolar macrophage," J. Appl. Physiol. vol. 60, no. 2, pp. 353-369, Feb. 1986.

[4] T. Ishida, K. E. Pinkerton, and M. Takeuchi, "Alveolar macrophage from cigarette smoke-exposed mice inhibits B lymphocyte proliferation stimulated with LPS," Respiration, vol. 77, no. 1, pp. 91-95, Jan. 2009

[5] L. G. Miller, G. Goldstein, M. Murphy, and L. C. Ginns, "Reversible alterations in immunoregulatory $\mathrm{T}$ cells in smoking. Analysis by monoclonal antibodies and flow cytometry," Chest, vol. 82, no. 5, pp. 526-529, Nov. 1982.

[6] M. Takeuchi, S. Nagai, and T. Izumi, "Effect of smoking on natural killer cell activity in the lung," Chest, vol. 94, no. 4, pp. 688-693, Oct. 1988.

[7] M. Ando, M. Sugimoto, R. Nishi, M. Suga, and S. Horio, "Surface morphology and function of human pulmonary alveolar macrophages from smokers and non-smokers," Thorax, vol. 39, no. 11, pp. 850-856, Nov. 1984.

[8] J. M. Löfdahl, J. Wahlström, and C. M. Sköld, "Different inflammatory cell pattern and macrophage phenotype in chronic obstructive pulmonary disease patients, smokers and non-smokers," Clin. Exp. Iimmunol., vol. 145, no. 3, pp. 428-437, Sep. 2006.

[9] O. C. Barriga and A. B. Rodríguez, "Decline in the phagocytic function of alveolar macrophages from mice exposed to cigarette smoke," Comp. Immunol. Microbial. Infect. Dis., vol. 17, no. 1, pp. 77-84, Feb. 1994.

[10] E. Ortega, F. Hueso, M. E. Collazos, M. I. Pedrera, C. Barriga, and A. B Rodríguez, "Phagocytosis of latex beads by alveolar macrophages from mice exposed to cigarette smoke," Comp. Immunol. Microbiol. Infect. Dis., vol. 15, no. 2, pp. 137-142, Apr. 1992.

[11] S. E. Barbour, K. Nakashima, J. B. Zhang, S. Tangada, and C. L. Hahn, "Tobacco and smoking: environmental factors that modify the host response (immune system) and have an impact on periodontal health,' Rev. Oral. Biol. Med., vol. 8, no. 4, pp. 437-460, Jan. 1997.

[12] M. Takeuchi, S. Nagai, A. Nakajima, M. Shinya, and C. Tsukano, "Inhibition of lung natural killer cell activity by smoking: the role of alveolar macrophages," Respiration, vol. 68, no. 3, pp. 262-267, Jan. 2001.

[13] T. Takeuchi, M. Nakajima, and K. Morimoto, "Relationship between the intracellular reactive oxygen species and the induction of oxidative
DNA damage in human neutrophil-like cells," Carcinogenesis, vol. 17, no. 8 , pp. $1543-1548$, Aug. 1996.

[14] T. Ishida, Y. Hirono, K. Yoshikawa, Y. Hutei, and M. Miyagawa, "Inhibition of immunological function mediated DNA damage of alveolar macrophages caused by cigarette smoke in mice," Inhal. Toxicol., vol. 21, no. 14, pp. 1229-1235, Dec. 2009.

[15] L. Wolz, G. Krause, and G. Scherer, "The comet assay with MCL-5 cells as an indicator of genotoxic treatment with chemicals and cigarette smoke condensates," Altern. Lab. Anim., vol. 30, no. 3, pp. 331-339, 2002.

[16] X. Liu, H. Conner, T. Kobayashi, H. Kim, and F. Wen, "Cigarette smoke extract induces DNA damage but not apoptosis in human bronchial epithelial cells," Am. J. Respir. Cell. Mol. Biol., vol. 33, no. 2 , pp. 121-129, Aug. 2005.

[17] H. Kim, X. Liu, T. Kobayashi, H. Conner, and T. Kohyama, "Reversible cigarette smoke extract-induced DNA damage in human lung fibroblasts," Am. J. Respir. Cell. Mol. Biol., vol. 31, no. 5, pp. 483-490, Nov. 2004.

Yuriko Hirono was born at Hyogo, Japan. She obtained her Bachelor of Engineering in 2007 and Master of Engineering in 2009 from Kyoto Sangyo University. Presently, she is working as research assistant in Kyoto Sangyo University. She has more than 10 publications. Her major filed is Immunology and Pulmonology.

Ayaka Kawazoe was born at Mie, Japan. She obtained her Bachelor of Engineering in 2010 and Master of Engineering in 2012 from Kyoto Sangyo University. Presently, she is working in MSD Co. Ltd. Her major filed Immunology and Pharmacology.

Masahito Nose was born at Kyoto, Japan. He obtained his Bachelor of Engineering in 2011 from Kyoto Sangyo University. Presently, he is studying as postgraduate student of Master Course in Kyoto Sangyo University.

Masaaki Sakura was born at Gunma, Japan. He obtained his Bachelor of Engineering in 1997 from Kyoto Sangyo University and Master of Science in 1999 from Osaka University. Presently, He is working as researcher in Hoyu Co., Ltd. He also belongs to Kyoto Sangyo University as visiting researcher for Immunology and Dermatology.

Minoru Takeuchi was born at March, 1951. He received DVM from Yamaguchi University in 1971 and PhD from Kyoto University in $1987 . \mathrm{He}$ worked as assistant professor in Kyoto University and belonged to UCDavis as visiting professor. Presently, he is working Kyoto Sangyo University as professor. His major filed is Immunology and Pulumonology. He has more than 80 publications. 